\title{
Quantum dynamics, dissipation, and asymmetry effects in quantum dot arrays
}

\author{
F. Rojas and E. Cota \\ Centro de Ciencias de la Materia Condensada - UNAM, Ensenada, Baja California, México 22800
}

S. E. Ulloa

Department of Physics and Astronomy, and Nanoscale and Quantum

Phenomena Institute, Ohio University, Athens, OH 45701-2979

(Dated: $7 / 2 / 02$ )

\begin{abstract}
We study the role of dissipation and structural defects on the time evolution of quantum dot arrays with mobile charges under external driving fields. These structures, proposed as quantum dot cellular automata, exhibit interesting quantum dynamics which we describe in terms of equations of motion for the density matrix. Using an open system approach, we study the role of asymmetries and the microscopic electron-phonon interaction on the general dynamical behavior of the charge distribution (polarization) of such systems. We find that the system response to the driving field is improved at low temperatures (and/or weak phonon coupling), before deteriorating as temperature and asymmetry increase. In addition to the study of the time evolution of polarization, we explore the linear entropy of the system in order to gain further insights into the competition between coherent evolution and dissipative processes.
\end{abstract}

PACS numbers: 73.21.La, 73.63.Kv, 85.35.Gv

\section{INTRODUCTION}

The size reduction of devices in microelectronics, and the fundamental limitations encountered or anticipated due to quantum mechanical effects, have turned attention to understanding nanometer scale structures. Systems where intrinsic quantum mechanical effects can play an important role and could even be used to advantage are under intense scrutiny. One recent prominent example of such systems are quantum dot cellular automata.

The original concept of "cellular automata" was introduced as $n$-dimensional arrays containing finite amplitude (information) per cell (or site) and connected to one another according to certain rules. This simple definition, however, governs the evolution of the entire array in rather complex ways. Quantum cellular automata are a natural generalization of this concept, where each site contains a quantum mechanical probability amplitude. In 1993, Lent and coworkers, 3 proposed the use of cellular automata architectures, composed of nanometer-scaled quantum devices (quantum dots) coupled through carefully chosen hopping and Coulomb interactions, to encode classical binary information in the different charge configurations of the system. The typical basic element in these quantum-dot cellular automata $(\mathrm{QCA})$ is a cell consisting of four quantum dots (QD) located at the vertices of a square and connected via tunneling barriers to their neighbors (see Fig. 11a). For two mobile electrons in each four-dot cell, Coulomb repulsion between the electrons causes the charge in the cell, in the ground state, to align along either of the two diagonals. The symmetrical arrangement of the system means that these two "polarization" states are degenerate and can be used to represent logic 0 and 1 as the bits in this system. The degeneracy is split by an appropriately designed driver field which allows control of the polarization of the ground state (and typically implemented as a second identical cell with externally controlled polarization). Tunneling barriers between dots are designed in such a way that intra-cell tunneling is possible, but inter-cell tunneling is not, and only Coulomb interaction from cell to cell is possible. Elemental cell designs have been experimentally realized using metal islands, and logic operations AND, OR and NOT have been implemented using arrays of such cells suitably arranged. $4 \mathrm{Fl}_{\mathrm{O}}$ Also, a clever implementation of QCA using magnetic elements has been recently reported

Although originally proposed as 'classical' and near dissipationless bit operators, QCA could in principle also be used to implement a quantum computer, as discussed recently $\mathrm{g}$ if the entire array system is coherent enough (and for sufficiently long times). In either the classical or quantum operation mode, QCA computers (as well as any other) should keep errors to a manageable limit. Of special significance in these systems, decoherence and dissipation are external sources of 'error' originating in the coupling between the system and its surroundings. Other aspects that have to be considered as possible sources of loss of control or desirable operation are imperfections in the dot fabrication, stray charged impurities, and the role of switching fields or drivers. It is then of interest to study the dynamical evolution of the QCA and monitor the charge density distribution over the basic cell elements under the influence of a driver cell, with emphasis on the effects of imperfections and dissipation. The charge configuration is conveniently monitored through Lent's cell polarization, 1 which is essentially a measure of the degree of alignment of the charges along the diagonals of the square cell:

$$
P=\frac{\rho_{1}+\rho_{3}-\left(\rho_{2}+\rho_{4}\right)}{\rho_{1}+\rho_{2}+\rho_{3}+\rho_{4}},
$$



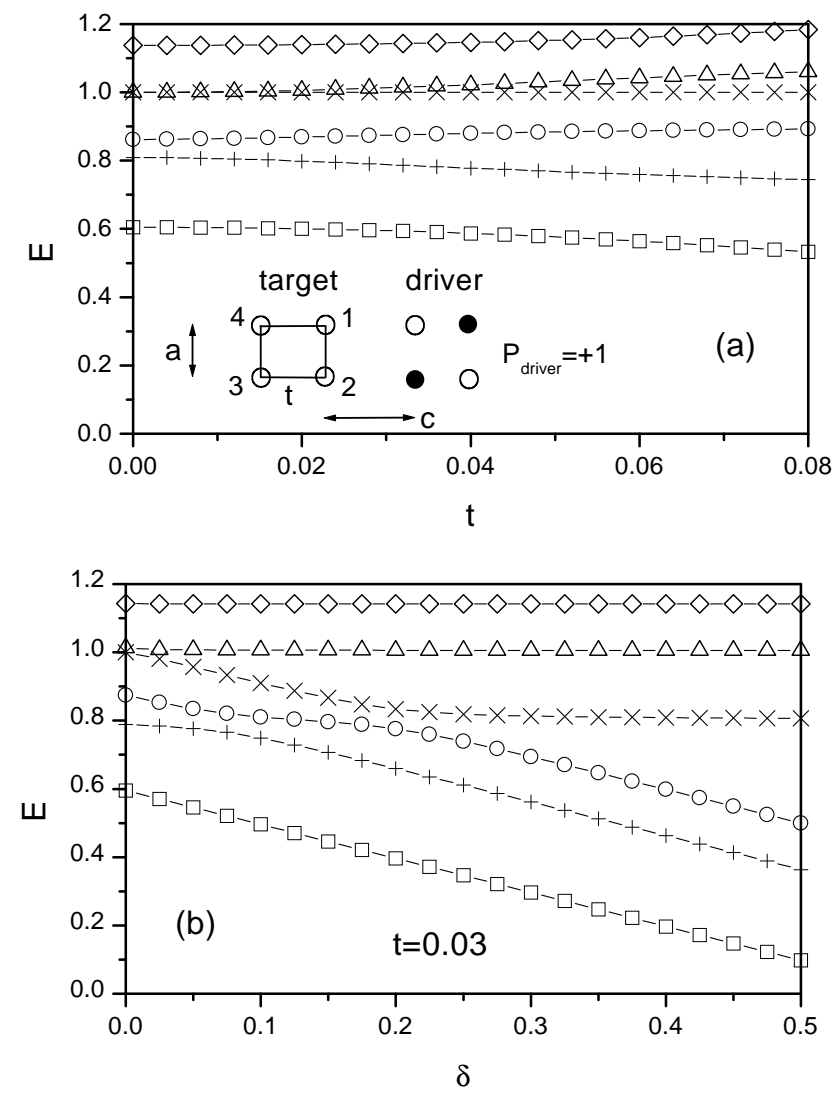

FIG. 1: (a) Energy level structure vs. $t$ for a target cell with driver at +1 . Energy in units of $V \simeq 1 \mathrm{meV}$, and $\delta=0$. Sketch at bottom: model QCA with four quantum dots per cell. Two charges are allowed to tunnel between nearest neighbors, with probability $t$. Driver cell on right indicates a state with polarization $P_{\text {driver }}=+1$. (b) Level structure vs. dot size asymmetry $\delta$. Notice anticrossings for $\delta \simeq 0.1$ and 0.2 in excited states. Separation to driver, $c=a$ in both panels.

where $\rho_{i}$ is the charge density at site $i$ (see Fig. 1a), and the numbering of the sites is in clockwise order. In previous works, the effect of dissipation on QCA has been included in the quantum equations of motion in a phenomenological way, 9 through a damping parameter. This model is shown to give qualitatively correct results only in the low temperature limit, as it will be evident in our calculations. Also, the temperature dependence of the cell polarization has been studied for the case of a cell composed of two Coulomb-coupled double dots, 10 and the contribution of relaxation processes to the speed of the cell response was discussed using stochastic equations for the population differences and dipole moments of the double dots. The effects of imperfections have been considered before, with 11.12313 and without14 dissipation, and found to have strong effects in the dynamical response, by delaying or slowing down the response of the target cell, and producing a less than optimal desired polarization. In this paper, we include the important effect of the environment through a microscopic model of electron-phonon interactions. The thermal bath is modelled with phonons in equilibrium at a given temperature and structural asymmetries are introduced via size variations of the quantum dots.

We should point out that in addition to their relevance as a new computational paradigm, QCA arrays provide a conceptually simple, yet intricate and subtle, quantum mechanical system in which to study fundamental coherence and dissipative processes. The parametrical requirements to make practical QCA devices yield a finite set of two-particle states which can be manipulated to yield insights into the loss of coherence in these and general quantum systems. As such, QCA provide then a model condensed matter system where dynamics and decoherence can be explored in detail. To this effect, we use a quantum open systems approach 1215 . 16 to study the dynamical evolution of the reduced density matrix $\hat{\rho}_{S}(t)$ of the system, and evaluate the competition between coherent (driver cell) and incoherent (thermal bath) field effects in the response of the basic cell. We study the effects of temperature, electron-phonon coupling intensity, and structural asymmetry on the behavior of the polarization as a function of time. In particular, we discuss the asymptotic polarization after switching events, and once the driver is in a stationary configuration, for different temperatures and structure parameters. An analysis of asymptotic fixed points in the resulting dynamics provides information on the system, as we will explicitly demonstrate and discuss later on.

A measure of the mixed character of a system described by a density matrix $\hat{\rho}$ is provided by the linear entropy, $s[\hat{\rho}]=\operatorname{Tr}\left\{\hat{\rho}-\hat{\rho}^{2}\right\} .17$ This quantity, which can be thought of as the lowest order approximation to the von Neumann entropy, is also called the idempotency defect or the purity of a state. Notice that the linear entropy is zero for a pure state, since $\operatorname{Tr}\{\hat{\rho}\}=\operatorname{Tr}\left\{\hat{\rho}^{2}\right\}=1$ in that case. Nonzero values of $s[\hat{\rho}]$ provide then a quantitative measure of non-purity of the state of a system. When monitored in time, the linear entropy provides a convenient measure of how fast the loss of quantum purity occurs in a system in contact with a bath, or the increase in coherence under appropriate 'pumping' conditions, as we will discuss below. Other uses of $s[\hat{\rho}]$ include the study of quantum manifestations of chaotic behavior in a complex system. 18 We explore the influence of temperature, electron-phonon coupling intensity and driver cell switching times on $s[\hat{\rho}]$, in order to understand the process of dissipation in the QCA system.

We find in all cases that high dissipation and asymmetry influence adversely the response of the basic cell, as one would intuitively anticipate. However, the behavior with temperature is quite subtle. For example, low temperature results in better switching and more faithful following of the driver by the target cell. Higher temperatures eventually produce quenching of the polarization and a dynamical evolution which basically ignores the 
driver. On the other hand, size defects in the dots result in lagging of the switching behavior with respect to the driver, and a faster switching window. This is produced by the pinning of a given polarization state by the energetics of the size defect, so that the driver potential has to apply a strong force before the target cell can follow. Similar lag and/or pinning, as well as sudden switch of the polarization is seen in the case of two target cells being driven by a single cell. A given cell in the middle of a chain would clearly have to deal with the influence of more than one cell, resulting in a more complex potential landscape and corresponding dynamical behavior. We also find for a single cell that in the case of low temperature or weak dissipation the cell recovers dynamical coherence, due to the 'pumping' of the driver, clearly overpowering the thermal bath. Other regimes will be discussed in detail.

The behavior of the polarization of the basic cell as a function of time when the polarization of the driver is fixed, as well as when it changes linearly, has been briefly discussed recently 12 There, we studied the symmetric (no defect) case, but taking account of dissipation in the system. In what follows, we present a full report of the QCA dynamical behavior under different electronphonon and temperature conditions, and analyze the linear entropy of the system during these processes.

The paper is organized as follows: in section II we describe the model for the entire system, composed of the QCA and the thermal bath. We use an extended Hubbard Hamiltonian to describe the Coulomb interactions present in the system (target cell(s) + driver cell). The thermal bath is represented by a set of harmonic oscillators, and the electron-phonon interaction enters as emission and absorption of phonons during electron tunneling events between quantum dots in the system. A Markovian approach to the dynamical evolution of the reduced density matrix is presented in II A, where the transition rates for the electron-phonon interaction in this system are explicitly obtained. In section III we present and discuss the behavior of the polarization and linear entropy, as function of relevant parameters, with special emphasis on effects of dissipation and asymmetry. Here, we also discuss the dynamics of multiple cells, as the QCA ultimate devices involve multiple cell geometries. Finally, we summarize and discuss our results in section IV.

\section{MODEL}

Consider a problem described by the Hamiltonian $\hat{H}=$ $\hat{H}_{S}+\hat{H}_{R}+\hat{V}_{S R}$, where the subscripts $S$ and $R$ stand for system and reservoir, respectively. In our case, $\hat{H}_{S}$ describes the target cell in the presence of a driver cell which provides external driving fields on the system. The interactions are introduced via an extended Hubbard Hamiltonian with both intra- and inter-cell Coulomb repulsion terms, as well as intra-cell tunneling,

$$
\begin{aligned}
\hat{H}_{S} & =\sum_{j} \epsilon_{j} \hat{n}_{j}-\sum_{<i j>}\left(t_{i j} \hat{c}_{i}^{\dagger} \hat{c}_{j}+c . c .\right) \\
& +\sum_{i>j} V_{i j} \hat{n}_{i} \hat{n}_{j}+\sum_{i, j} W_{i j} n_{i}^{d}(t) \hat{n}_{j} .
\end{aligned}
$$

The first three terms describe the target or active cell and the last term describes the Coulomb interaction between the electrons in the target cell and a driver cell which is externally controlled. Here, $\epsilon_{j}$ is the confinement energy level in the $j^{\text {th }}$ quantum dot (QD) in the target cell. In this term, possible dot asymmetries or local potential variations are introduced by changing the energy in a given $k^{t h} \mathrm{QD}, \epsilon_{k} \rightarrow \epsilon-\delta$, where $\delta$ is the measure of the imperfection due to different dot size or a change in the local environment, and $\epsilon$ is the energy of the other (identical) dots. The amplitude $t_{i j}$ is the tunneling matrix element between nearest neighbors $\langle i j\rangle$ on the same cell, $\hat{c}_{j}^{\dagger}$ and $\hat{c}_{j}$ are creation and annihilation operators for site $j, \hat{n}_{j}=\hat{c}_{j}^{\dagger} \hat{c}_{j}$ is the electron number operator at site $j, V_{i j}$ is the Coulomb interaction between sites $i$ and $j$ in the target cell, and $W_{i j}$ is that between site $i$ in the driver cell (with charge density $n_{i}^{d}(t)$, which in general changes in time) and site $j$ in the target cell. A generalization to multiple cells is straightforwardly given by inter-cell interactions. 14 Following the notation of Lent et al. 3 the distance between nearest-neighbor dots (length of the square side) is $a$, while the separation between the target and driver cells, located side by side, is $c$ (see inset Fig. 1a). Notice we consider spinless electrons, although considering two-particle states with given total spin is also possible in our formalism.

The Hamiltonian for the reservoir is given by a set of harmonic oscillators of frequency $\omega_{k}, \hat{H}_{R}=\sum_{k} \hbar \omega_{k} \hat{b}_{k}^{\dagger} \hat{b}_{k}$, where $\hat{b}_{k}^{\dagger}\left(\hat{b}_{k}\right)$ are creation (annihilation) operators of phonons. Finally, the electron-phonon interaction is given by a general expression,

$$
\hat{V}_{S R}=\sum_{k} \sum_{<i j>} \alpha_{k i j} \hat{c}_{i}^{\dagger} \hat{c}_{j}\left(\hat{b}_{k}^{\dagger}+\hat{b}_{k}\right),
$$

corresponding to emission/absorption of a phonon for electron tunneling events and with matrix elements given by the coefficients $\alpha_{k i j}$. Notice that the physics of the phonon interactions in the system is well described by the model chosen here. Although phonons are in principle present everywhere, and not only during tunneling events, they only provide an 'on-site' energy renormalization/shift and broadening, which will be ignored here, as it yields a uniform correction to the entire cell. In contrast, the phonon-assisted tunneling events described by $V_{S R}$ affect significantly the dynamics of the cell, as they provide an effective coupling mechanism among the different levels in the spectrum and allow for energy relaxation processes.

We assume, as in [19], that the quantum dots have a characteristic size $d \simeq 50 \mathrm{~nm}$ defined on a GaAs/AlGaAs 
heterostructure, with effective mass $m^{*}=0.067$. The typical distance between nearest-neighbor dots is $a \gtrsim$ $100 \mathrm{~nm}$, and the dielectric constant of the medium is $\approx$ 12. These values give an estimate of the Coulomb repulsion between nearest neighbor dots of $V \approx 1 \mathrm{meV}$, which is taken as the unit of energy in this paper. One should notice that the first excited single-particle state in each dot lies at quite a high energy, $\Delta \approx\left(\hbar^{2} / 2 m^{*}\right)(2 \pi / d)^{2} \approx$ $9 \mathrm{meV} \gg V$, so that one can safely consider a single orbital per site, although additional single-particle levels in each dot could in principle be included. Notice also that the on-site Coulomb energy is quite high and prevents the double occupation of the dot, so that our basis set of states ignores that possibility. We should comment that successful implementations of QCA elements using metallic islands are in a multiple-orbital regime, since in that case $\Delta \ll V$ for typical $1 \mu \mathrm{m}$-size islands. The condition of overall electrical neutrality of the target (and driver) cell is included in the calculation by distributing a uniform positive background charge $+2 e$ throughout the cell $(e / 2$ in each dot), which results in multipolar fields from/at each cell.

Notice that we study the behavior of the target cell interacting with the driver via Coulomb interaction only (no inter-cell tunneling allowed). This interaction is described by the last term in the Hamiltonian $H_{S}$, Eq. (2), and assumed to be time dependent via $n_{i}^{d}(t)$. For a fixed driver and no dissipation, the solution can be found by direct numerical diagonalization of $H_{S}$. In that case, the effect of various parameters on the polarization have been studied to show that the energy spectrum is strongly influenced by even small defects, which affect the bistability of the cell.20 In the case of a time-dependent driver, we have to take into account that the driver promotes transitions to intermediate excited states. In fact, the dynamical competition between the driver (coherent terms in the equation of motion) and the bath (dissipative terms) causes a non-trivial time evolution of the system. We have studied different 'driving schemes' but will mostly consider here the case of a driver with a linear switching of its polarization, $P_{\text {driver }}(t)=1-2 t / \tau$, for times $0 \leq t \leq \tau$, where $\tau$ is the switching time. This corresponds to the configuration of charge densities in the driver cell of $n_{1}^{d}(t)=n_{3}^{d}(t)=1-t / \tau$ and $n_{2}^{d}(t)=n_{4}^{d}(t)=t / \tau$. For the four-dot cell arranged on a square, we take $V_{i j}=V$ for nearest-neighbor dots, while all others scale with their (inverse) separation. We also set all $t_{i j}$ equal to a constant $t$ between nearest neighbors only (not to be confused with time).

\section{A. Markovian master equation}

In the interaction picture $(I)$, the evolution of the total density matrix is given by

$$
\begin{aligned}
\frac{\partial \hat{\rho}^{(I)}(t)}{\partial t} & =-\frac{i}{\hbar}\left[\hat{V}_{S R}^{(I)}(t), \hat{\rho}^{(I)}(0)\right] \\
& -\frac{1}{\hbar^{2}} \int_{0}^{t} d t^{\prime}\left[\hat{V}_{S R}^{(I)}(t),\left[\hat{V}_{S R}^{(I)}\left(t^{\prime}\right), \hat{\rho}^{(I)}\left(t^{\prime}\right)\right]\right]
\end{aligned}
$$

where $\hat{V}_{S R}^{(I)}$ is the cell-bath interaction operator, and the expansion is valid up to second order in $\hat{V}_{S R}^{(I)} 16$

Our approach uses a closed and time-local equation for the reduced density matrix (RDM): $\hat{\rho}_{S}^{(I)}(t)=$ $\operatorname{Tr}_{R}\left\{\hat{\rho}^{(I)}(t)\right\}$ where the trace is carried out over the degrees of freedom of the reservoir, $R$. The following fundamental assumptions are made in this approach: (1) the system $S$ and reservoir $R$ are initially uncorrelated, which implies $\hat{\rho}(0)=\hat{\rho}_{S}(0) \hat{\rho}_{R}(0)=\hat{\rho}^{(I)}(0) ;(2)$ a stationary reservoir at temperature $T$ with equilibrium density matrix $\hat{\rho}_{R}(0)=\exp \left(-\hat{H}_{R} / k_{B} T\right) / \operatorname{Tr}\left\{\exp \left(-\hat{H}_{R} / k_{B} T\right)\right\}$, exists at all times; and (3) the correlation time $\tau_{c}$ in the reservoir is much shorter than characteristic times for the RDM system to change appreciably, meaning that $\hat{\rho}_{S}^{(I)}\left(t^{\prime}\right) \approx \hat{\rho}_{S}^{(I)}(t)$, if $t^{\prime}-t \lesssim \tau_{c}$, while the state of the reservoir at time $t^{\prime}$ has already no correlation with the state at time $t$ (Markov approximation). Thus one arrives at the time evolution equation for the RDM elements (details of the derivation can be found in the literature 12 ), which in the Schrödinger picture is given by

$$
\dot{\hat{\rho}}_{S}(t)_{s s^{\prime}}=-i \omega_{s s^{\prime}} \hat{\rho}_{S}(t)_{s s^{\prime}}+\sum_{m n} \tilde{R}_{s s^{\prime} m n} \hat{\rho}_{S}(t)_{m n}
$$

The first term on the right represents reversible dynamics ("coherent" effects), in terms of the transition frequencies $\omega_{s s^{\prime}}=\left(E_{s}-E_{s^{\prime}}\right) / \hbar$, while the second term describes relaxation (irreversible dynamics). In this part, the relaxation tensor $\tilde{R}_{s s^{\prime} m n}$ can be written explicitly as

$$
\tilde{R}_{s s^{\prime} m n}= \begin{cases}\delta_{n m}\left(1-\delta_{m s}\right) \tilde{W}_{s m}-\delta_{m s} \delta_{n s} \sum_{k \neq s} \tilde{W}_{k s}, & \left(s=s^{\prime}\right) \\ -\gamma_{s s^{\prime}} \delta_{m s} \delta_{n s^{\prime}}, & \left(s \neq s^{\prime}\right)\end{cases}
$$

Here, $\tilde{W}_{n m}$ are transition rates from state $|m\rangle$ to $|n\rangle$, which in terms of the properties of bath and target cell, can be expressed as

$$
\begin{aligned}
\tilde{W}_{m n} & =\frac{2 \pi}{\hbar^{2}} D^{2}\left|g\left(\omega_{m n}\right)\right|^{2} \mathcal{D}\left(\omega_{m n}\right)\left\{\left|S_{n m}\right|^{2} \bar{n}\left(\omega_{m n}\right)\right. \\
& \left.+\left|S_{m n}\right|^{2}\left[1+\bar{n}\left(\omega_{m n}\right)\right]\right\}
\end{aligned}
$$


where $S_{m k}$ are matrix elements of the electronic part of the target cell interacting with the phonon reservoir, Eq. (3)); $\bar{n}(\omega)=\left\langle\hat{b}^{\dagger}(\omega) \hat{b}(\omega)\right\rangle=\left(e^{\beta \hbar \omega}-1\right)^{-1}$ is the average phonon number in the reservoir at temperature $T=1 / k_{B} \beta$. The first term $(\propto \bar{n})$ describes the absorption of phonons, while the second $(\propto \bar{n}+1)$ describes phonon emission, both at a frequency given by the system's transition energy $\omega_{m n}$. Notice that the matrix elements $S_{m n}$ embody the appropriate selection rules for the different states involved in the transitions. For simplicity, we consider a model where the amplitude of interaction does not depend on the electronic states of the target cell, and has the form $\alpha_{k i j}=D g_{k}$, where $D$ is the constant prefactor of the electron-phonon interaction and is determined from the deformation potential model 21 We should emphasize that this model introduces dissipation effects only at finite temperature, and cannot account for elastic decoherence effects.22 Here, $g_{k}=g\left(\omega_{k}\right) \sim$ $\omega_{k}^{1 / 2}$ describes the frequency dependent amplitude of the electron-phonon interaction in the deformation potential model; $\mathcal{D}(\omega) \sim \omega^{2}$ is the density of states in a Debye model and $\omega_{m n}$ is the transition frequency between states $|m\rangle$ and $|n\rangle$. It is clear that other phonon channels with different dispersion relations and/or matrix elements exist, but we believe that other modes would not be as effective in introducing dissipation in this system (e.g., optical phonons occur at energies too high to couple effectively), nor qualitatively change the results discussed here. In Eq. (6), $\gamma_{s s^{\prime}}$ is a so-called non-adiabatic parameter whose real part gives a contribution to the time decay of the off-diagonal density matrix elements, and is then directly responsible for the loss of coherence. These parameters can be written in terms of the transition rates by $\operatorname{Re} \gamma_{s s^{\prime}}=\left(\sum_{k \neq s} \tilde{W}_{k s}+\sum_{k \neq s^{\prime}} \tilde{W}_{k s^{\prime}}\right) / 2$. The imaginary part of $\gamma_{s s^{\prime}}$ is an intrinsic relaxation rate for each transition, which we assume negligible, $\operatorname{Im} \gamma_{s s^{\prime}}=0$, as it cpuld be incorporated in the transition frequencies, $\omega_{s s^{\prime}} .16$

It is interesting to point out that Eq. (5), written for the diagonal elements, takes the form:

$$
\dot{\hat{\rho}}_{S}(t)_{s s}=\sum_{m} \tilde{W}_{s m} \hat{\rho}_{S}(t)_{m m}-\hat{\rho}_{S}(t)_{s s} \sum_{m} \tilde{W}_{m s}
$$

which has a simple interpretation: the probability of finding the level $|s\rangle$ occupied at time $t, \hat{\rho}_{S}(t)_{s s}$, increases due to transitions from all other levels $|m\rangle$ to $|s\rangle$ (first term) and decreases due to transitions from $|s\rangle$ to all other levels $|m\rangle$ (second term).

Note also that the condition $\dot{\hat{\rho}}_{S}(t)_{s s^{\prime}}=0$ in Eq. (5) corresponds to 'fixed points' of the dynamics of the system, as both the terms related to coherence, $\left(\hat{\rho}_{S}\right)_{s s^{\prime}}$ for $s \neq s^{\prime}$, as well as the diagonal terms which determine the population in each state, do not change in time. These fixed point values depend only on the properties of the transition rates and the energy spectrum of the system (as the external drive reaches a stationary configuration), and provide a direct determination of the dissipation times produced by the thermal bath. It can be seen from Eq.
(7) that the transition rates satisfy

$$
\frac{\tilde{W}_{n m}}{\tilde{W}_{m n}}=\exp \left(-\frac{\hbar \omega_{n m}}{k_{B} T}\right),
$$

which guarantees the detailed balance condition. It is important to emphasize that we have derived an explicit expression for the transition rates $\tilde{W}_{m n}$, which would behave at low temperatures, $T \rightarrow 0$, and/or low dissipation (small $D^{2}$ ) as the form used by Zozoulenko et al., in a qualitative way.11 Notice however that the normalization condition in 11] is an added requirement in their phenomenological approach, while it is included naturally in our treatment. We should also mention that the detailed balance ratio in (9) will determine the fixed point values of $\hat{\rho}_{S}$ and all the corresponding physical properties, including the different state populations. Since these will in general be asymmetric, the polarization value of the QCA is reduced, and as we will see later, this results also in a limiting value for the linear entropy. A detailed analysis of the asymptotic fixed points in terms of the structure parameters will also shed light into the dynamical evolution of the system, as we will discuss.

The solution of the master equation (5) for the time evolution of the RDM is carried out numerically using a Runge-Kutta fourth order algorithm. We ensure, by a proper choice of the integration step, that the general normalization property $\operatorname{Tr}\left\{\hat{\rho}_{S}\right\}=1$ for the RDM is satisfied at all times. Notice that an instantaneous basis is incorporated in the calculation to take into account the evolution of the ground state induced by the time-dependent driver configuration. The basis functions are then defined by the eigenvalue/eigenvector problem of the coherent Hamiltonian of the target cell, $\hat{H}_{S}(t)|m\rangle=E_{m}^{S}(t)|m\rangle$, for a given time $t$ while the driver is in the process of switching, and the instantaneous energy in the target cell is $E_{m}^{S}(t)$. Notice also that the characteristic time variation of the driver $\tau$ is long compared with the target-cell's natural frequencies $\omega_{l l^{\prime}}$, so that $\tau \gg \hbar / \omega_{l l^{\prime}}$. This is effectively the requirement for an adiabatic switching of the driver. As is clear in Fig. 1, the characteristic frequencies of the system are $\omega_{l l^{\prime}} \lesssim 1 \mathrm{meV}$, so that $\hbar / \omega_{l l^{\prime}} \gtrsim 1$ ps. This would require $\tau$ to be a few nanoseconds for the adiabatic switching regime to be valid.

The numerical solution of the RDM is used to calculate the dynamics of the polarization in the target cell, as the physical observable of interest here. The dynamical behavior of $P(t)$, calculated from the charge density in each site as $\rho_{i}(t)=\operatorname{Tr}\left\{\hat{\rho}_{S}(t) \hat{n}_{i}\right\}$, and of the linear entropy $s\left[\hat{\rho}_{S}\right]$, are then determined for a given set of parameters. In order to get better insights in the problem, we also carry out a calculation of the fully coherent dynamics, ignoring the phonon thermal bath (setting $D=0$ in Eq. 7). In the coherent limit, the relevant parameters for the dynamical evolution are those defining the structure, including the tunneling amplitude $t$, the separation of target and driver cell $c$, the switching time of the linear driver $\tau$, and the size of the dot asymmetry $\delta$, if any. For the dissipative problem, on the other hand, it is clear that 
in addition to the structure features, the important parameters are the temperature of the thermal bath $T$, and the amplitude of the electron-phonon interaction $D$. Of course, the phonon density of states, as well as the interaction details, will also play a role in the evolution of the system. Our model parameters provide an adequate and realistic description of these systems in semiconducting QCA.21

\section{RESULTS AND DISCUSSION}

Figure 1 1 a shows the level spectrum in the target cell for a fixed driver polarization, $P_{\text {driver }}=1$, as shown in the inset. We see that the four-fold symmetry of the structure and consequent degeneracy is broken by the driver, even for $t=0.20$ Moreover, increasing the intra-cell tunneling $t$ produces additional level splittings, as one would expect. We should point out that the polarization of the ground state follows that of the driver, $P_{\text {cell }}^{\text {grnd }} \simeq 1$, but increasing hopping $t$ reduces that value somewhat. The first excited state has $P_{\text {cell }}^{e x c} \simeq-1$, and is separated from the ground state by a gap of $\simeq 0.2$ in the range shown (our energy units are $V \simeq 1 \mathrm{meV}$ ). The four higher states have vanishing polarization, as the charges are located on neighboring sites along the sides of the square. The basic operation of the QCA requires adiabatic evolution from a polarized ground state to the other with opposite polarization. Transitions to higher excited states allow for non-adiabatic terms which yield to depolarization of the target cell. Figure 1 $1 \mathrm{~b}$ shows the influence of a "defect" in dot size. Here, the dot at site 1 has an energy lower than the others by an amount $\delta$ (corresponding to a larger dot). Increasing this asymmetry produces a strong shift of the spectrum, and perhaps most important for QCA operation, a change of the polarization of the first excited state. This is apparent in the anticrossing seen in $1 \mathrm{~b}$ bor $\delta \simeq 0.1$, where an opposite polarization state takes over as the first excited state.20 The defect then will produce a pinning of the polarization when operating the QCA if the $\delta$ is small, and even prevent the switching of the target cell altogether if $\delta$ is large.

When the polarization of the driver cell changes linearly with time, the polarization of the target cell evolves as well, as presented in Fig. 2a for the non-dissipative case, and for different temperatures. One can see that the polarization of the target cell follows the driver very well, and switches smoothly over a short time window $(\approx \tau / 5)$ centered about the time when $P_{\text {driver }}=0$. This general behavior is not affected for different values of the switching time $\tau$ we tested (over a widg range within the adiabatic regime; results not shown).23 We see that the response of the target cell deteriorates for increasing temperature. At low temperature, $T=T_{1} \simeq 1 \mathrm{~K}$, the target cell has a slightly decreased polarization after the driver is fully switched, and the switching time of the cell is not greatly affected. However, for $T=5 T_{1}$, the target cell polarization is quenched after $0.5 \mathrm{~ns}$ and ignores even a fully
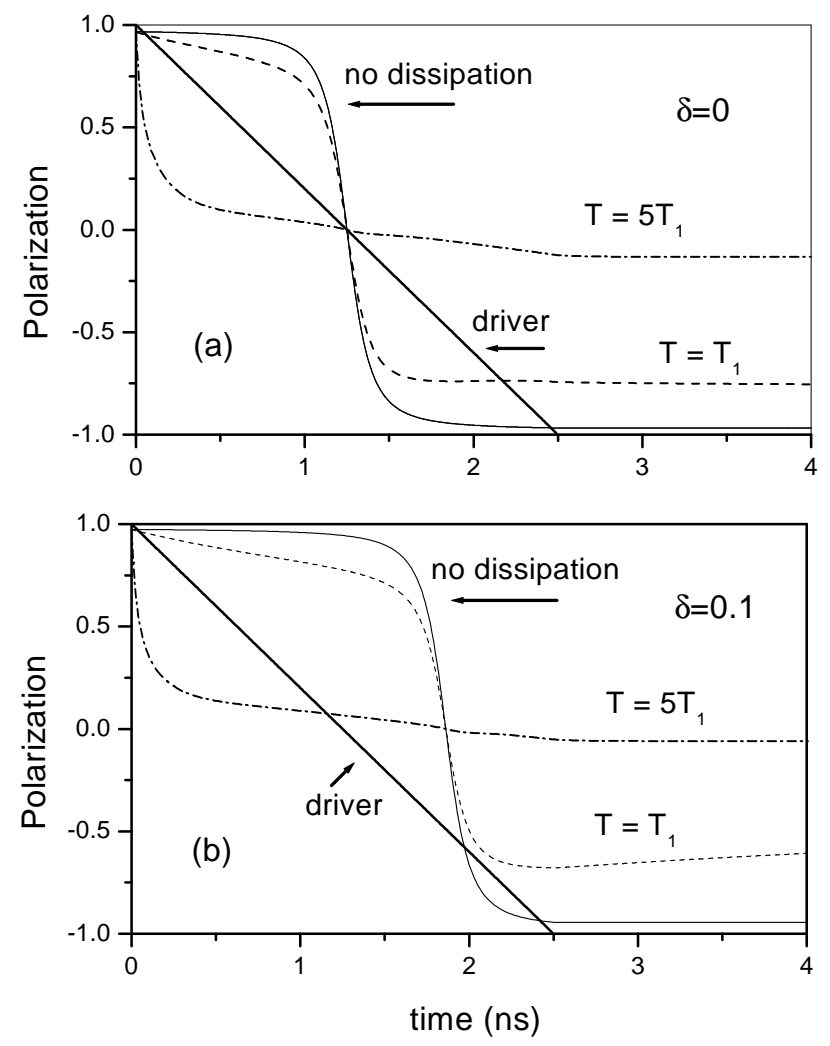

FIG. 2: Time evolution of target cell polarization as driver cell switches linearly from $P_{\text {driver }}=+1$ to -1 in a time $\tau=$ $2.5 \mathrm{~ns}$, for different temperatures. (a) Symmetric cell with four identical dots $(\delta=0)$. (b) Asymmetric cell with one lower energy dot, $\delta=0.1$, at site 1 . In all plots, $t=0.03$ and $D^{2}=0.05$.

polarized driver. [Notice that the natural unit of temperature in this problem is the difference in energy between the first and second excited states, when the driver is fixed at $P_{\text {driver }}=1$ (Fig. 17p), as this transition has one of the smallest natural frequencies $\omega_{l l^{\prime}}$, and it produces depolarization of the cell, as discussed above. In particular, we take as characteristic its value for $t=0.03$ in the symmetric case, $T_{1}=0.0862 \approx 1 \mathrm{~K}$. Temperatures are compared to this natural scale in the problem.] The deterioration of the response of the cell with temperature is consistent with Eq. (9) where the different rates at low temperature would favor transitions to the ground state, while as temperature increases, transitions up and down become equally probable. This results in more symmetric level populations, more equal electron probabilities in all dots, and consequently the depolarization of the target cell. It is somewhat surprising that even this low temperature would yield such strong quenching of the QCA polarizability (a value which of course depends also on how strong $D$ is in Eq. 7). Figure $2 \mathrm{~b}$ shows an exam- 

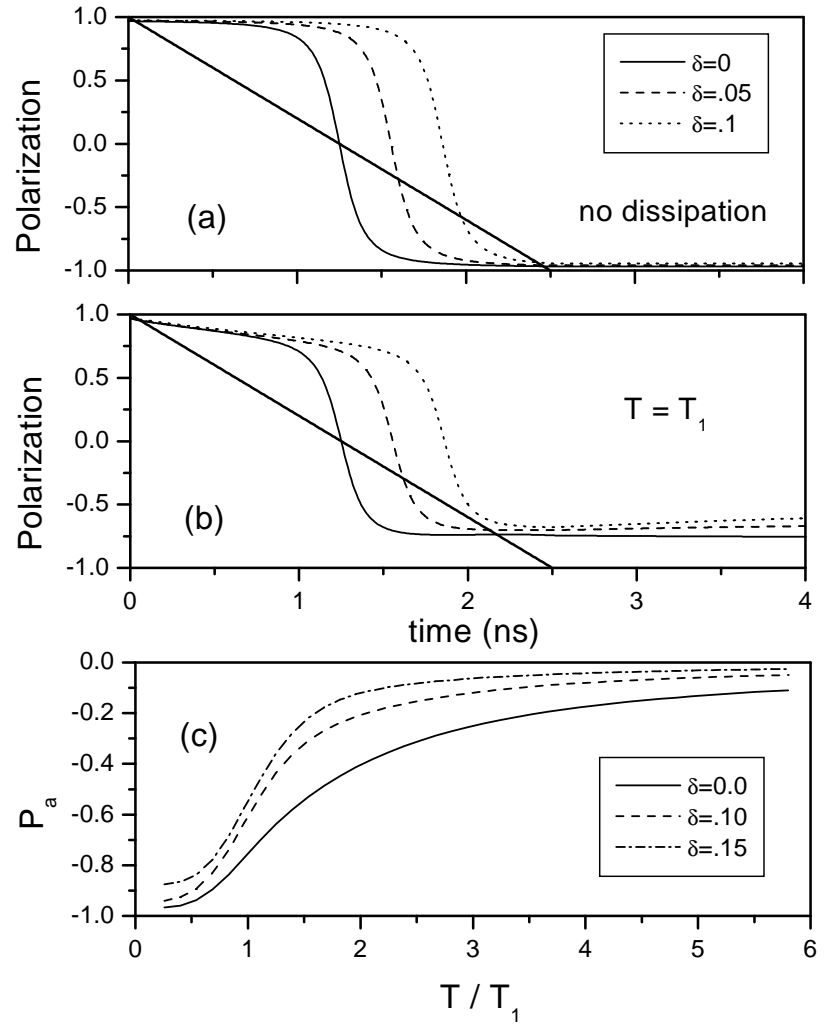

FIG. 3: Time evolution of target cell polarization with a linear switch drive, $\tau=2.5 \mathrm{~ns}$, for different asymmetry $\delta$. (a) No dissipation $(D=0)$; (b) at temperature $T=T_{1}$. Notice target cell lagging the switch of the driver increases with $\delta$. (c) Long-time asymptotic polarization, $P_{a}$ vs. temperature $T$ for different $\delta$ values. In all panels, $t=0.03$ and $D^{2}=0.05$.

ple of the effects of asymmetry (quantum dot at site 1 has lower energy than the rest), for the same parameter values and temperatures as in Fig. 2a. We observe that at low temperatures, the response of the target cell lags behind the driver, although the switching time is not affected substantially. However, the target cell reaches asymptotic values of polarization which are smaller (in absolute value) than in the ideal symmetric case. In this case too, changing $\tau$ over a wide range does not affect this lagging behavior. It is evident that for $T=5 T_{1}$, the target cell is almost completely depolarized, despite the "help" of the larger dot which pins the polarization of the ground and excited states to $\simeq+1$ (and induces the lag seen at low temperatures).

Figure 3 shows more of the effects of asymmetry and temperature on the response of the target cell. Figure 3 a shows the results for no dissipation, and panel (b) those for $T=T_{1}$. From these figures, we see again that the important effect of asymmetry is to produce a lag or delay in the response of the target cell as asymmetry in-

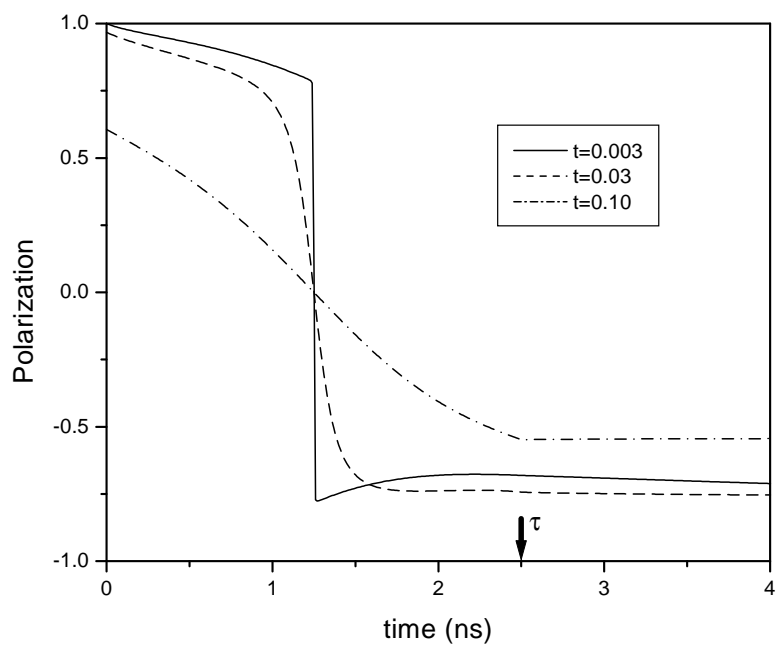

FIG. 4: Time evolution of polarization in a linear switch driver, $\tau=2.5 \mathrm{~ns}$ (see arrow). Increasing tunneling between dots first improves $(t=0.03)$ and then deteriorates $(t=0.1)$ the final polarization. $D^{2}=0.05, T=T_{1}$, and $\delta=0$ in all plots.

creases. On the other hand, the effect of temperature is to decrease the asymptotic polarization in absolute value, associated with the delocalization of electrons within the cell. Notice also in Fig. $3 \mathrm{~b}$ that for finite $T$, the polarization of the target cell continues evolving in time (deteriorating), even after the driver is fully polarized. In all cases, the polarization reaches asymptotic values $P_{a}$ (long after the driver has stopped changing) which are a manifestation of 'fixed points' of the system dynamics for the quantum dot populations. These long-time values of the polarization depend on temperature (and indirectly on structure parameters and energy levels) as shown in Fig. 3c, for different asymmetries. The nearly full depolarization of the target cell at high temperature is evident and anticipated. What is somewhat counterintuitive is the fact that the depolarization increases with asymmetry, as the pinning that yields the lag assists now in the depolarization by favoring the population of the states with the larger dot.

Figure 1 shows the effects of tunneling coupling intensity $t$ on the time evolution of the polarization for a symmetric cell system $(\delta=0)$. We see that increasing hopping between dots first introduces a smoother transition following the linear driver $(\tau=2.5 \mathrm{~ns})$ and a better polarization. Increasing $t$ however, delocalizes the charges in the quantum dots and reduces the polarization of the target cell, as one can see from the long time limits in the figure. In order to look more closely at the non-monotonic behavior of $P_{a}$, we analyze this quantity as a function of the tunneling coupling $t$ in Fig. Fa. We find that, for $T=T_{1}, P_{a}$ attains a minimum value at $t \approx 0.02$. From this figure we can see that the 

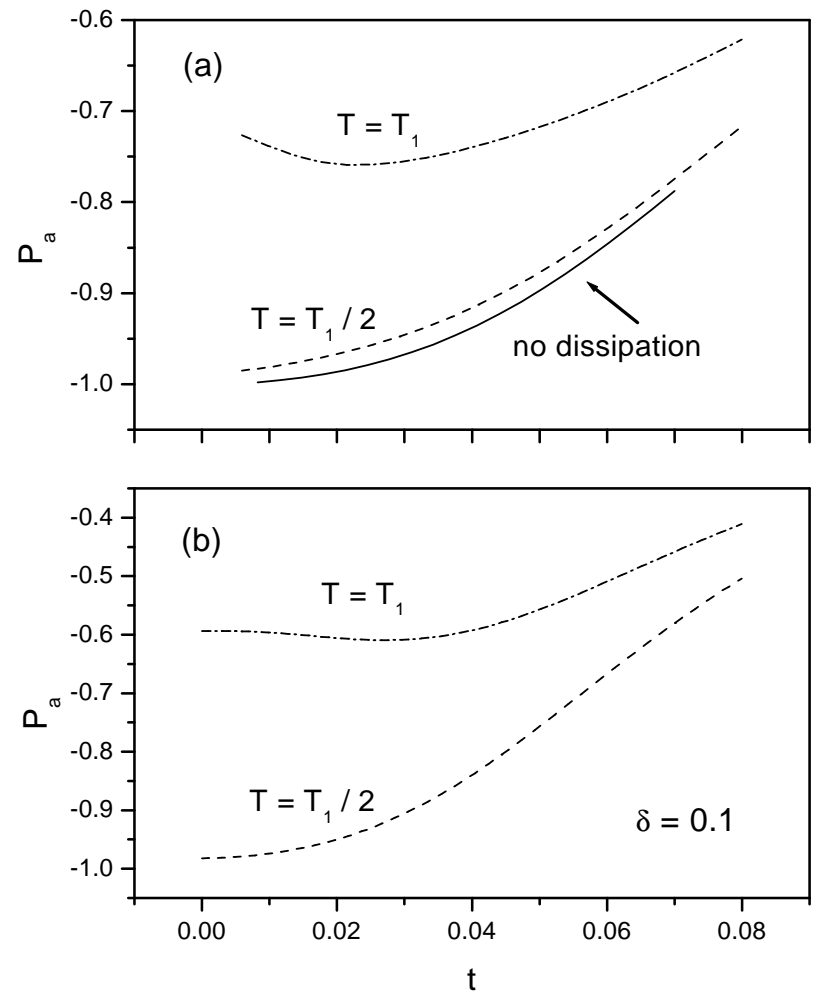

FIG. 5: Long-time asymptotic polarization $P_{a}$ vs. hopping $t$ at different temperatures, as in Fig. T. The non-monotonic behavior is seen only for $T \approx T_{1}$. (a) Symmetric cell $(\delta=0)$; (b) asymmetric case, $\delta=0.1$. In both panels, $D^{2}=0.05$.

drop in polarization at small values of $t$ is due to dissipation processes: We expect that at low $t$ values the electrons would tend to be well localized at the corners of the cell (ground state), giving $P= \pm 1$, depending on the driver. This is indeed what is observed at low temperatures. However, as temperatures increases, depolarization transitions from the ground state to excited states become more likely. As $t$ increases substantially, the polarization decreases (in absolute value) due to the delocalizing effects of both tunneling and dissipation, as expected. However, for $t \lesssim 0.02$, the possibility of transitions which in fact populate the ground state increases, and therefore improve slightly the asymptotic polarization of the system. Qualitatively similar results are obtained in the asymmetric case (Fig. 5b), although with lower absolute values of the asymptotic polarization in this case, as discussed above (Fig. 2 and 3), and a shallower oscillation.

We have also studied the dynamics of two target cells and driver in line (Fig. 6). There are a number of interesting features in this case. We notice first that the switching for both target cells lags behind the driver con- siderably, as the cells switch only after $P_{\text {driver }} \gtrsim 75 \%$. Moreover, the switch occurs over a much shorter time scale than for the single target cell $(\simeq \tau / 25)$. This change is understandable if one thinks that the target cell closest to the driver (cell 1) is in essence between two cells with different polarization and has its wrong polarization somewhat pinned (this is behavior also seen in other work 11 ). Increasing temperature to $T=T_{1}$ makes the switching a bit smoother although still with a sudden change, and with not as good final polarization. Notice in particular that the asymptotic polarization of target cell 2 is poorer than that of cell 1 . As the temperature increases to $T=2 T_{1}$, the target cells barely follow the drive, and the polarization of cell 2 is basically zero at long times. It is clear that control of the tunneling process as the "signal" is transmitted down the line would be essential in proper operation of QCA arrays. Such "clocked switching" has been discussed by Lent and comprkers as a solution to steer cells into better compliance.24 Coherent simulations of such steering 14 as well as experiments in metallic QCA25 verify that this is a good operating scheme.

Knowing the time evolution of the density matrix we can also calculate the linear entropy of the QCA system $s\left[\hat{\rho}_{S}\right]$, to monitor the degree of non-purity introduced during the switching process by the thermal bath. In Fig. 7 we show the results for different temperatures, for the case of weak and strong electron-phonon interactions, as measured by the value of $D^{2}$ in Eq. (7). As in previous cases, the polarization of the driver cell is switched linearly from +1 to -1 in a time $\tau=2.5$ ns. In Fig. Za, the case of 'weak' electron-phonon coupling, $D^{2}=0.05$, we see that at low temperatures $s\left[\hat{\rho}_{S}\right]$ increases monotonically until it reaches a final stable value. It is interesting to see that as $P_{\text {driver }}=0$ or $P_{\text {driver }}=-1$ (at 1.25 and $2.5 \mathrm{~ns}$, respectively), one can see kinks in the entropy curve, indicating the switch off of driving forces. The long time asymptotic value increases (coherence decreases) with increasing temperature, as one would expect. However, at still higher temperatures and/or for strong electron-phonon coupling (Fig. $7 \mathrm{~b}$ ), $s\left[\hat{\rho}_{S}\right]$ first increases and then decreases. In these cases, we see a maximum in the linear entropy curve at $\approx \tau / 2$, when the polarization of the driver cell goes through zero. Notice in fact that whenever $P_{\text {driver }} \simeq 0$, the level spectrum in the target cell shows a great deal of degeneracies. As the driver turns negative (for time after $\tau / 2$ ) the splittings are reinstated and the effect of the driver seems to be one of a coherent 'pump' which produces a drop in the purity, even as dissipative transitions are present. In other words, the thermal dissipation seems to decrease with respect to the coherent transitions induced by the driver, producing a drop in the linear entropy of the system. After the driver is stationary, after time $\tau$, the thermal transitions quickly produce the steady state in the density matrix (or level populations) given by the detailed balance equation (77). For stronger phonon coupling, $D^{2}=0.5$, and high temperatures (Fig. $7 \mathrm{~b}$ ), the 

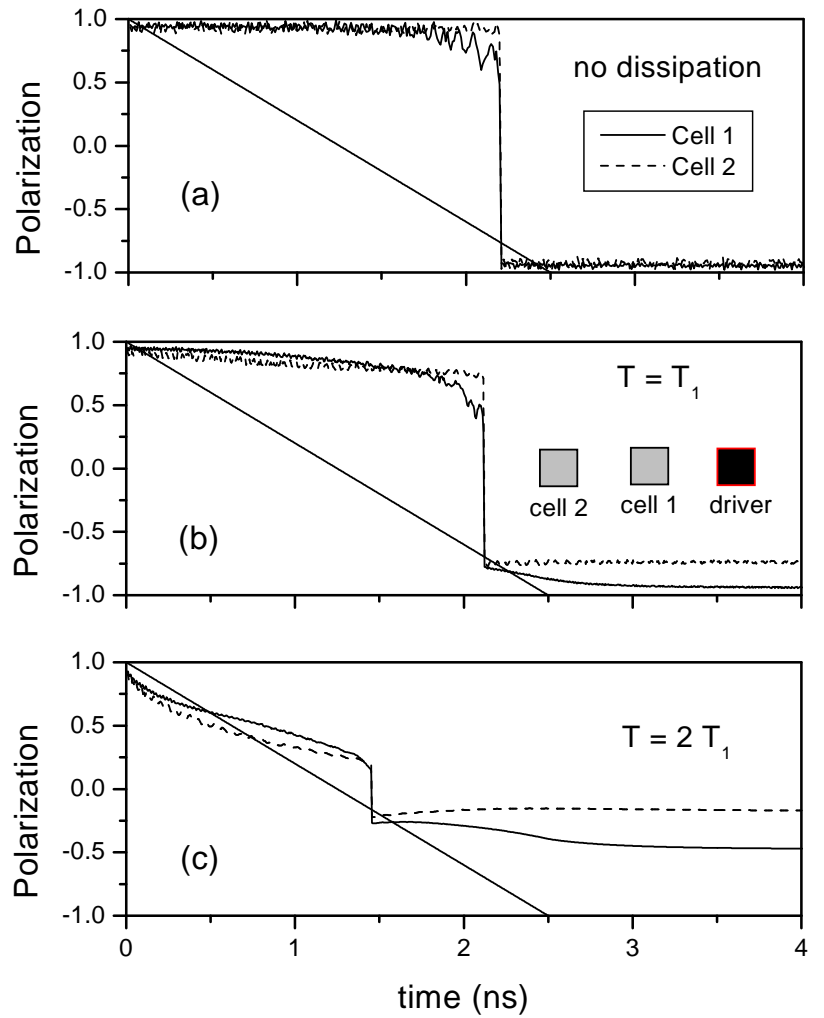

FIG. 6: Polarization of two active cells and linear driver, as shown in inset in panel (b). (a) Coherent dynamics; (b) at $T=T_{1} ;$ (c) at $T=2 T_{1}$. Notice cells lag the driver, except at higher temperatures. More distant cell (2) switches more suddenly than cell 1 , and has smaller $P_{a}$. In all plots, $t=0.03$ and $D^{2}=0.05$.

purity of the system deteriorates further and the driver is not able to 'push' the system back into a more coherent state. Notice that the state of 'maximal delocalization' would be achieved as $\hat{\rho}_{\text {Sii }} \simeq 1 / n$, where $n$ is the size of the basis, so that $\operatorname{Tr}\left\{\hat{\rho}^{2}\right\} \simeq 1 / n$. In that case, one would obtain $s \simeq 1-1 / n$, giving $5 / 6$ in our case. This value is still higher than the asymptotic value of $s$ for the highest temperatures in Fig. $7 \mathrm{~b}$, so that even then the system is not 'fully' decoherent.

Let us explore this competition between dissipation and coherent evolution in more detail. Since $\operatorname{Tr}\left\{\hat{\rho}_{S}\right\}=1$ is a general property of the density matrix in any representation, one can instead investigate the behavior of $\operatorname{Tr}\left\{\hat{\rho}_{S}^{2}\right\}=1-s\left[\hat{\rho}_{S}\right]$ directly as a function of the relevant parameters.26 Notice in fact that this trace has two contributions

$$
\begin{aligned}
\operatorname{Tr} \hat{\rho}_{S}^{2} & =\sum_{i}\left(\hat{\rho}_{S}^{2}\right)_{i i} \\
& =\sum_{i}\left(\hat{\rho}_{S i i}\right)^{2}+\sum_{i \neq j}\left|\hat{\rho}_{S i j}\right|^{2}
\end{aligned}
$$
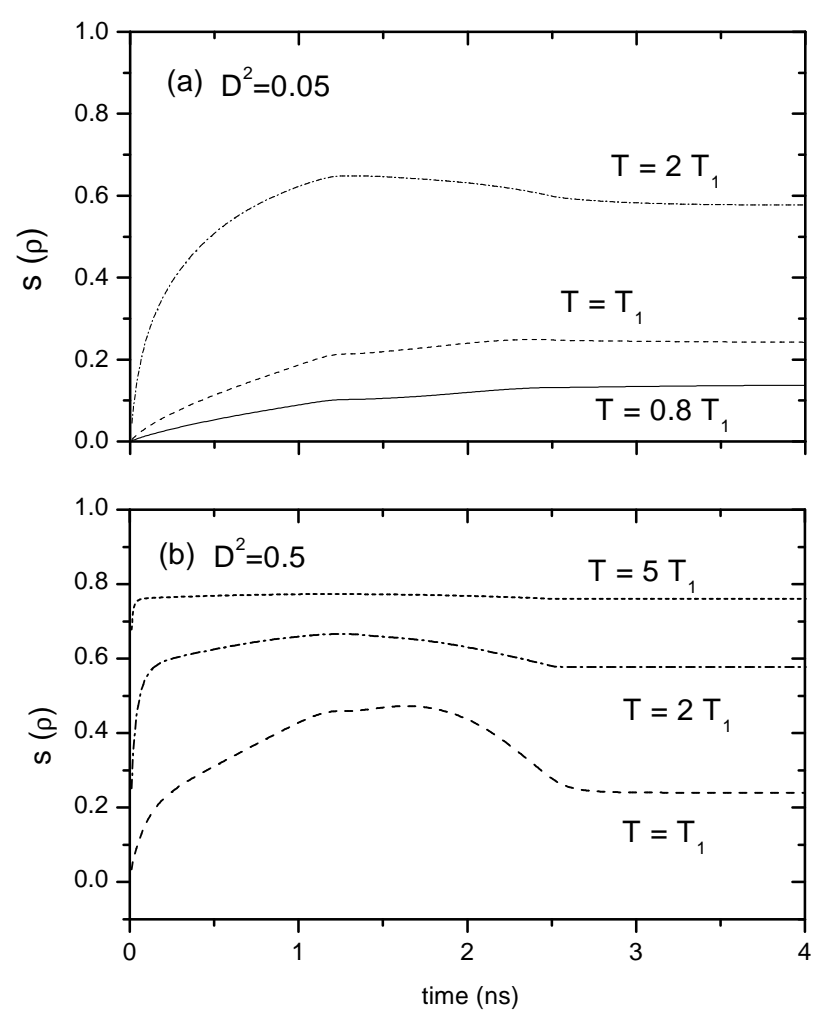

FIG. 7: Time evolution of linear entropy or purity of the system in a linear switch, $\tau=2.5 \mathrm{~ns}$ at different temperatures. (a) Weak coupling, $D^{2}=0.05, t=0.03$; (b) strong coupling, $D^{2}=0.5, t=0.03$. Notice entropy $d r o p$ after $\tau / 2$ at intermediate dissipation.

namely, the diagonal and non-diagonal elements of the density matrix. Comparing the two terms as a function of time, for example, would give one further insights of the overall complex processes, since the behavior of the non-diagonal elements gives information on the evolution of coherence in the system. Figure 8 shows the trace as function of time for two values of the temperature, explicitly showing the contributions from the diagonal and nondiagonal elements of $\hat{\rho}_{S}$. We see in Fig. 8a an increase in the contribution from the non-diagonal ("coherent") elements, with maximum at $\tau / 2$, exactly compensated by a decrease in the contribution from the diagonal elements, as the driver and the bath are promoting transitions. Notice also that the values of the diagonal components before and after $\tau / 2$ are approximately the same (with a similar behavior for the non-diagonal terms). Thus for low temperatures $T=T_{1} / 10$ (panel a), the sum remains approximately constant, equal to 1 , and the system is close to the pure limit with $s \approx 0$. However, for $T=T_{1}$, we see that this sum decreases with time, due essentially to the corresponding decrease in the diagonal elements. 


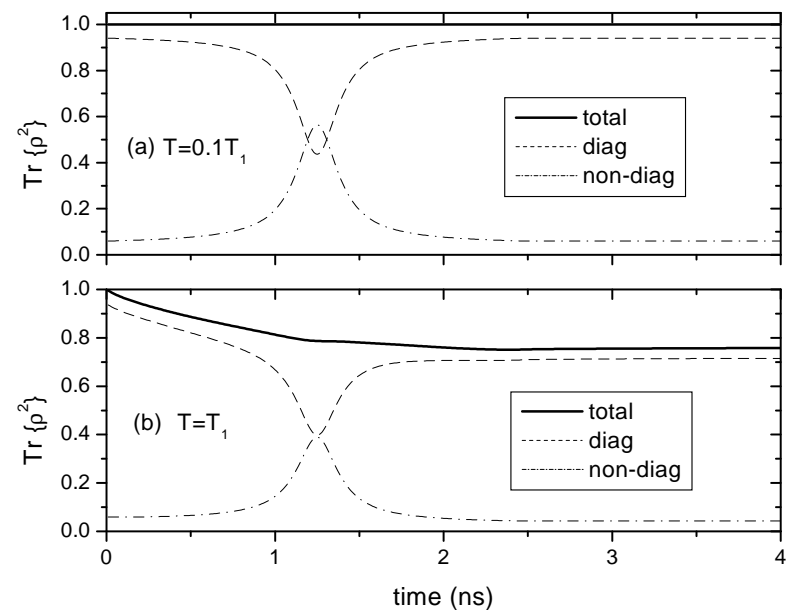

FIG. 8: Trace of $\hat{\rho}^{2}$ vs. time during a linear switch of the driver cell, $\tau=2.5 \mathrm{~ns}$, and different temperatures. (a) $T=T_{1} / 10$, (b) $T=T_{1}$. Both diagonal and non-diagonal sums are shown, as well as the total trace, as in Eq. 10. In both panels, $t=0.03$ and $D^{2}=0.05$.

Notice that as the driver switches and 'pumps' the target cell system, the off-diagonal coherent terms contribute less to the sum as the temperature increases (its peak value is smaller in (b) than in (a); so that the system is less coherent indeed), and their contribution for longer times is lower than at the start of the switch (time zero). This gradual decay in the coherent contribution shows even more substantially (and less intuitively) in the diagonal terms, which one would expect to be less affected perhaps. Since $\operatorname{Tr}\{\hat{\rho}\}=1$, the drop in the diagonal terms of $\operatorname{Tr}\left\{\hat{\rho}^{2}\right\}$ means that the population of each level $\left(\hat{\rho}_{\text {Sii }}\right)$ has been re-distributed and made somewhat more equal (although still much higher than the 'maximal delocalization' state discussed above with $\operatorname{Tr}\left\{\hat{\rho}^{2}\right\} \simeq 1 / 6$ ).

It is also interesting to investigate the influence of switching time and of the function controlling the switching. Figure 9 shows results for $\operatorname{Tr}\left\{\hat{\rho}_{S}^{2}\right\}$ for a smoother driver transition. In this case, the driver has no rapid off/onsets and the evolution of $P_{\text {driver }}$ is more adiabatic near the ends of the sweep than before (see panel c). Panel (b) shows the trace at low temperatures, which shows a similar rise (drop) in non-diagonal (diagonal) density matrix elements as in Fig. Ba. However, as the time during which $P_{\text {driver }} \simeq 0$ is shorter now (effectively larger slope in panel c), the window of variation is also reduced. Higher temperature in panel 9 a shows also a narrower time window variation, but an identical amplitude as in Fig. 8b. Other runs with different driver switching slopes follow this behavior too: narrower windows for larger slopes near $P_{\text {driver }} \simeq 0$ (not shown). This would suggest that the amplitude variation of the diagonal/offdiagonal components is only a function of temperature, while the time window over which they vary is a function
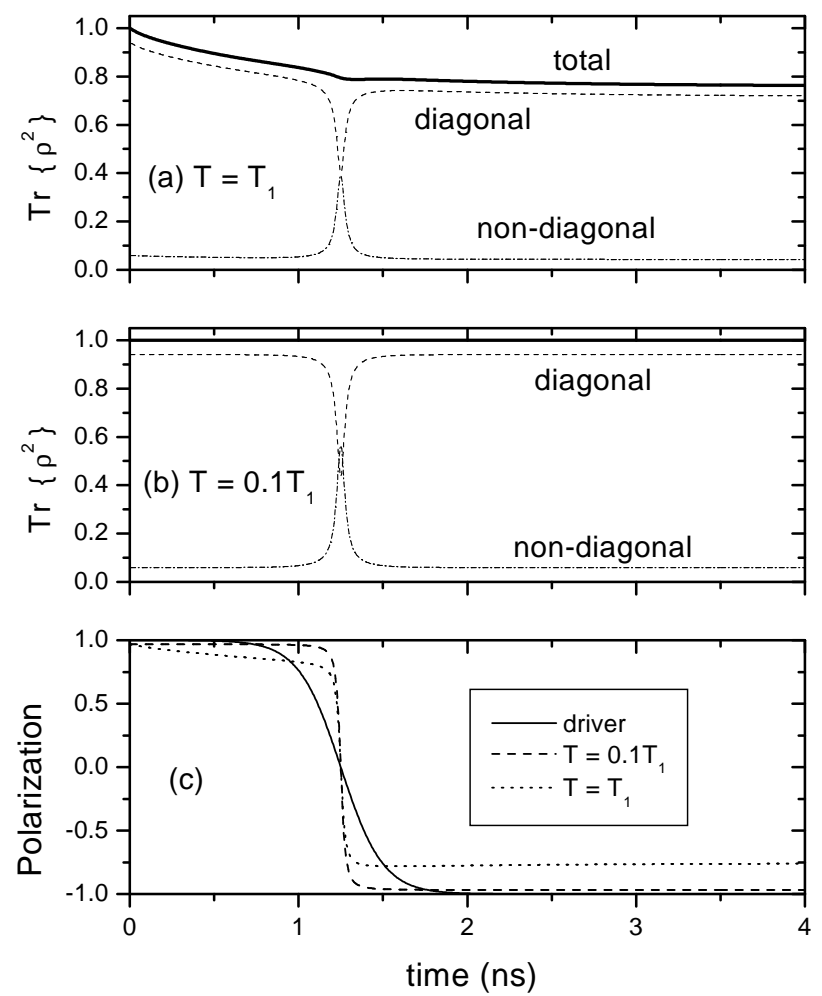

FIG. 9: Time evolution for a smoother driver switch. (a) Trace of $\hat{\rho}_{S}^{2}$ at intermediate temperature, $T=T_{1}$, showing diagonal and non-diagonal contributions. (b) Trace contributions for $T=T_{1} / 10$. (c) Driver (solid curve) and target cell polarizations for different temperatures. In all panels, $t=0.03$ and $D^{2}=0.05$.

of how long the driver is close to zero polarization.

\section{CONCLUSIONS}

We have studied the behavior of the polarization of a square array of quantum dots with two mobile electrons. The quantum dynamical evolution in response to a driver cell whose polarization is changing with time in a prescribed manner, has been shown to depend strongly on switching or driver characteristics, symmetry of the cell and on temperature. Our results show that the asymptotic values of the polarization in a stationary driver are fixed points of the system that change with temperature, tunneling probabilities and imperfections, but not on driving schemes. The optimal response of the QCA arrays is affected adversely by temperature and imperfections, with a strong tendency toward depolarization as temperature or asymmetry increase. Our calculations indicate that good control of the QCA is only achieved for 
temperatures of at most a few kelvins. From our two target cell results, it is likely that "clocked switching" would be required for faithful operation of a chain, so that the polarization information is not degraded down the line. We have also analyzed dissipation effects on the response of the basic cell through calculation of the linear entropy or purity of the system. We find that the study of this quantity is a good tool in furthering the understanding of a quantum system as relatively simple, but still quite subtle, as the QCA architecture. Apart from providing insights as a function of time and driver characteristics, the entropy also shows clearly that at high temperatures, the basic cell is no longer in the required/desired final state but in one where the polarization tends to zero and maximizes the entropy.

\section{Acknowledgments}

This work was supported in part by CONACYT Project 27702-E. SEU is supported in part by US DOE grant no. DE-FG02-91ER45334. We acknowledge support from the Condensed Matter and Surface Sciences Program at Ohio University.
1 S. Wolfram, Rev. Mod. Phys. 55, 642 (1983).

2 G. Grössing and A. Zeilinger, Complex Syst. 2, 197 (1988); 2, 611 (1988).

3 C. S. Lent, P. D. Toughaw, and W. Porod, J. Appl. Phys 74, 3550 (1993).

4 A. O. Orlov, I. Amlani, G. H. Bernstein, C. S. Lent and G. L. Snider, Science 277, 928 (1997).

5 I. Amlani, A. Orlov, G. Toth, G. H. Bernestein, C. S. Lent and G. L. Snider, Science 284, 289 (1999).

${ }^{6}$ G. L. Snider, A. O. Orlov, I. Amlani, X. Zuo, G. H. Bernstein, C. S. Lent, J. L. Merz and W. Porod, J. of Appl. Phys., 85, 4283 (1999)

7 R. P. Cowburn and M. E. Welland, Science 287, 1466 (2000).

8 G. Toth and C. S. Lent, Phys. Rev. A 63, 052315 (2001).

${ }^{9}$ C. K. Wang, I. I. Yakimenko, I. V. Zozoulenko and K. F. Berggren, J. Appl. Phys. 84, 2684 (1998), and references therein.

10 A. Yu. Smirnov, N. J. M. Horing and L. G. Mourokh, J. Appl. Phys. 87, 4525 (2000).

11 I. I. Yakimenko, I. V. Zozoulenko, C.-K. Wang and K.-F. Berggren, J. Appl. Phys. 85, 6571 (1999).

12 E. Cota, F. Rojas and S. E. Ulloa, Mat. Res. Soc. Symp. Proc. Vol. 642, paper J1.12 (2000), eds. R. Leon, S. Fafard, D. Huffaker and R. Notzel.

13 F. Rojas, E. Cota and S. E. Ulloa, phys. stat. sol. (b) 230, 377 (2002).

14 F. Rojas, E. Cota and S. E. Ulloa Physica E 6, 428 (2000).

15 H. B. Sun and G. J. Milburn Phys. Rev. B 59, 10748 (1999).

16 G. Mahler and V. A. Weberru $\beta$, Quantum Networks: Dynamics of Open Nanostructures (Springer-Verlag, BerlinHeidelberg, 1995).

17 W. H. Zurek, S. Habib, and J. P. Paz, Phys. Rev. Lett. 70,
1187 (1993).

18 K. Furuya, M.C. Nemes and G. Q. Pellegrino, Phys. Rev. Lett., 80, 5524 (1998).

19 C. S. Lent and P. D. Tougaw, J. Appl. Phys. 75, 1818 (1994).

20 F. Ramírez, E. Cota and S. E. Ulloa, Phys. Rev. B 62, 1912 (2000)

21 The value of $D$ in Eq. (7) depends on the deformation potential of the semiconductor, as we consider coupling to acoustic phonons. Typical values of 1 to $3 \mathrm{eV}$ yield a value of $D^{2} \approx 7$ to 20 in our units. As the potential confinement of the electrons in the dot yields poorer coupling to acoustic phonons in the solid with characteristic long wavelengths, the effective value of $D$ is likely much smaller. We anticipate that the value of $D$ is smaller by a factor of $\approx 5$, and then use a value of $D^{2}=0.5$ as our 'strong coupling' regime.

22 A. O. Caldeira and A. J. Leggett, Phys. Rev. Lett. 46, 211 (1981); 48, 1571 (1982).

23 In all the time evolution plots shown, the initial condition of the target cell is chosen as the ground state of the system at the given hopping $t$, for $P_{\text {driver }}=+1$. This yields a smooth non-oscillatory oscillations at the start of the driver switchings. Other initial configurations with polarization similar to the ground state yield time evolutions with similar characteristics in $P$ and in linear entropy.

24 C. S. Lent and P. D. Tougaw, Proc. IEEE 85, 541 (1997); G. Toth and C. S. Lent, J. Appl. Phys. 85, 2977 (1999).

25 A. O. Orlov, I. Amlani, R. K. Kummamuru, R. Ramasubramanianm, G. Toth, C. S. Lent, G. H. Bernstein, and G. L. Snider, Appl. Phys. Lett. 77, 295 (2000).

${ }^{26}$ K. Blum, Density Matrix Theory and Applications (Plenum Press, NY, 1981). 